\title{
A Novel Approach to Systematic Switching Control Design for a Class of Hybrid Systems
}

Peter V. Zhivoglyadov and Richard H. Middleton

Department of Electrical and Computer Engineering

The University of Newcastle, N.S.W. 2308 Australia

email address: eepeter; rick@ee.newcastle.edu.au

\begin{abstract}
In this paper we propose and investigate a new systematic switching control design method applicable to a class of nonlinear discrete time hybrid systems.
\end{abstract}

\section{Introduction}

The main objective of this paper is to present a systematic algebraic approach to stabilizability analysis for a class of nonlinear discrete time hybrid systems. The novelty of the adopted approach lies in the fact that unlike conventional nonlinear control the control burden is explicitly shifted to a logical level thus creating the need for the development of new analysis/design methods.

\section{Problem Statement}

We consider the following class of nonlinear discrete time hybrid systems.

High Level Discrete Event Dynamics:

$$
\mathbf{S}=\{\mathbf{Q}, \boldsymbol{\Sigma}, \delta, \phi, \Delta \mathbf{T}, \boldsymbol{\Omega}\}
$$

Low Level Continuous State Dynamics:

$$
x(t+1)=f(x(t), i(x), \xi(t))
$$

where $\mathbf{S}$ is a finite state machine with $Q$ and $\Sigma$ being finite sets of symbolic states and events, respectively; $\Sigma=\Sigma_{c} \cup \Sigma_{u n} \neq \varnothing, \Sigma_{c}$ and $\Sigma_{u n}$ denote the sets of controlled and uncontrolled events, respectively. $\delta: Q \times \Sigma \rightarrow Q$ is a partial transition function. Equivalently the finite state machine $\mathbf{S}$ can be presented as a directed graph whose nodes represent discrete states and whose edges correspond to transitions. Each state $\xi \in Q$ of the automaton $\mathbf{S}$ is interpreted as an activity (phase) of finite length $\Delta t(\xi) \in \Delta T(\xi) \subset \mathrm{N}_{+}$while a transition $\xi_{i} \rightarrow \xi_{j}, \forall \xi_{i}, \xi_{j} \in Q$ enabled by the event $\sigma_{i j} \in \Sigma$ is instantaneous. A triple $e_{i j}=\left\{\xi_{i}, \sigma_{i j}, \xi_{j}\right\}$ such that $\xi_{i}, \xi_{j} \in Q$ and $\delta\left(\xi_{i}, \sigma_{i j}\right)$ ! will be referred to as a transition from the discrete state $\xi_{i}$ to $\xi_{j}$ enabled by the event $\sigma_{i j}$. The set $\Omega \subset \mathbf{R}^{\mathbf{n}}$ further referred to as the normal operating domain, reflects continuous specifications clarified later. Equation (2.2) describes the low level continuous dynamics (LLD) with $x$ denoting the continuous state of the low level subsystem (2.2) and $i(x)$ and $\xi(t)$ being the uncontrolled and controlled (respectively) switching indices affecting the evolution of the continuous dynamics. In this paper we will use the notion of a cell partition [1] defined on a compact set $\Omega \subset \mathbf{R}^{\mathbf{n}}$, representing continu- 
ous states specifications. For each discrete state $\xi \in Q$ the set $\Omega$ is presented as a finite union of convex polytopes $\Omega=\bigcup_{i=1}^{L} \Omega_{i}, \Omega_{i} \subset \mathbf{R}^{\mathbf{n}}, \forall i=1, \ldots, L$. Within each cell (assuming that $\xi$ is fixed) the dynamics are $x(t+1)=f(x(t), i, \xi)$ where $f: \mathbf{R}^{\mathbf{n}} \times \mathbf{N}_{+} \rightarrow \mathbf{R}^{\mathbf{n}}$. Thus, the indices $i$ and $\xi$ are responsible for the changes in the dynamics of the hybrid system caused by the evolution of the continuous and discrete states, respectively. The high level switching controller is defined as a mapping $\Gamma(\xi, x): Q \times \mathbf{R}^{\mathbf{n}} \rightarrow \mathbf{2}^{\boldsymbol{\Sigma}_{\mathrm{c}}}$. In the sequel we use the following assumptions: (A1) $0 \in$ int $\Omega$; (A2) Each cell $\Omega_{i}, i=1,2, \ldots, L$ is a convex polytope in $\mathbf{R}^{\mathbf{n}}$; (A3) The cells are non-overlapping, that is, $\left\{\Omega_{i}-\partial \Omega_{i}\right\} \cap\left\{\Omega_{j}-\partial \Omega_{j}\right\}=\emptyset, \forall i, j, i \neq j ;(\mathbf{A 4})$ For any $x \in \Omega_{i} \cap \Omega_{j} \neq \emptyset, \forall i, j=1, \ldots, L$ and $\xi \in Q$ the low level continuous dynamics are defined as $x(t+1) \in\{x(t+1): x(t+1)=f(x(t), l, \xi), l \in\{i, j\}\}$ (A5) $\Sigma=\Sigma_{c}=\Sigma_{f c}$ where $\Sigma_{f c}$ stands for a set of forced transitions; (A6) For every pair $\left\{\xi_{i}, \xi_{j}\right\}$ there exists a transition $e_{i j}=\left\{\xi_{i}, \sigma_{i j}, \xi_{j}\right\}$.

Assumption (A4) guarantees the uniqueness of the solutions of the equation (2.2) within each cell while the uniqueness of the solutions on the boundary of two or several cells is not assumed. Assumptions (A5),(A6) define an unconstrained hybrid system in which every discrete transition is admissible, controlled and forcible. Since our main objective is a control design method the description of the hybrid system explicitly showing only controlled transitions appears to be convenient. An example of such a system would be a multi-switch hybrid system depicted in Figure 1 where $\mathbf{W}_{\mathbf{i}}$ represent electrical components, and $\mathbf{K}_{\mathbf{i}}$ represent switches. For every possible combinations of binary switches the hybrid system evolves as a cell partition based one.

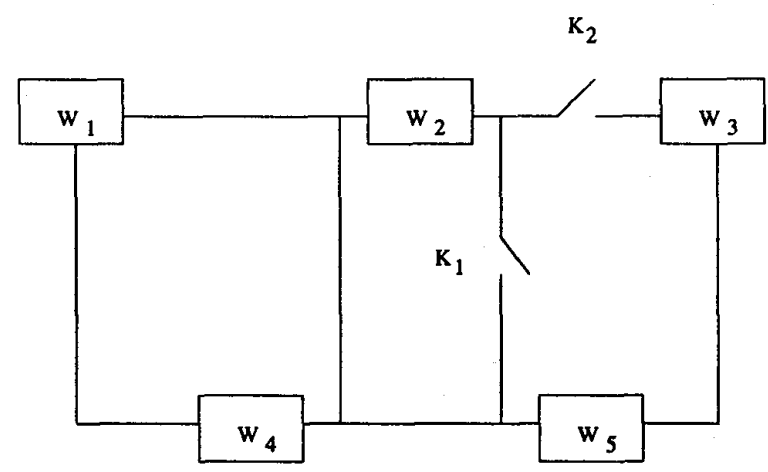

Figure 1: Multi-switch Hybrid System

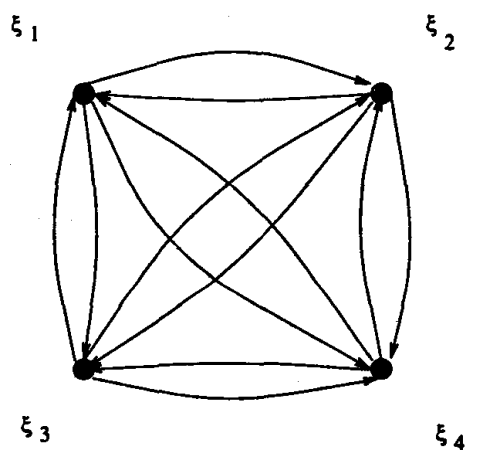

Figure 2: Multi-switch Hybrid System

Definition 2.1 A hybrid system (2.1),(2.2) controlled by a switching controllers $\Gamma(\xi, x)$ is said to be $\Omega$-stable if the following conditions are satisfied: (1) $x(t) \in \Omega$ for all $t \geq t_{0}, t_{0} \in \mathbf{N}_{+}$, and $x\left(t_{0}\right) \in \Omega$, (2) $\lim _{t \rightarrow \infty}\|x(t)\|=0$, (3) The low level continuous subsystem is stable in the sense of Lyapunov.

The notion of $\Omega$-stabilizability in the class switching controllers $\mathcal{L}: \Gamma(\xi, x)$ follows naturally from the previous definition. The three major steps outlined below are essential in the proposed method. 


\section{Step 1. Discrete Event Composition}

The main idea behind this procedure is superposition of the cell partitions associated with each discrete mode. This would allows us to obtain a cell partition based description of the system where the evolution of the continuous dynamics within each cell depends on the chosen switching sequence.

Before we proceed further we recall that an arbitrary convex polytope $\Omega_{j}(\xi)$ can be constructed as the intersection of $p(\xi)$ linear half-spaces, each given by an affine inequality $c_{j l}(\xi) x \geq z_{j l}(\xi), l=1,2, \ldots, p(\xi)$. Given decompositions of the normal operating domain $\Omega=\bigcup_{i=1}^{L(\xi)} \Omega_{i}(\xi), \forall \xi \in Q$ associated with each discrete state $\xi \in Q$ we define a discrete event composition of the system as $\Omega=\bigcup_{i=1}^{J} \Omega_{i}$ with each cell $\Omega_{i}$, $i=1, \ldots, J$ satisfying the conditions: (C1) Each $\Omega_{i}$, $i=1, \ldots, J$ is a convex polyhedron, (C2) For every hyperplane $H \in\left\{H_{j l \xi}\right\}$

$$
H_{j l \xi}=\left\{x: c_{j l}(\xi) x=z_{j l}(\xi), 1 \leq l<p(\xi), \xi \in Q\right\}
$$

and every $\Omega_{j}$ the following relation holds

$$
H \bigcap \Omega_{j} \neq \emptyset \Leftrightarrow H \bigcap \Omega_{j} \in \partial\left\{\Omega_{j}\right\}
$$

where $\partial\{\cdot\}$ stands for the boundary of a set, (C3) For each face, $\mathbf{F}_{i}, i=1, \ldots, s$ of $\Omega_{j}$ there exists a hyperplane $H$ from the set (3.3) such that $\mathbf{F}_{i} \in H$.

This is illustrated in Figures 3,4. The geometry of the cell partitions of a two discrete state hybrid system is depicted in Figure 3 while the resulting cell partition satisfying conditions (C1)-(C3) is given in Figure 4.

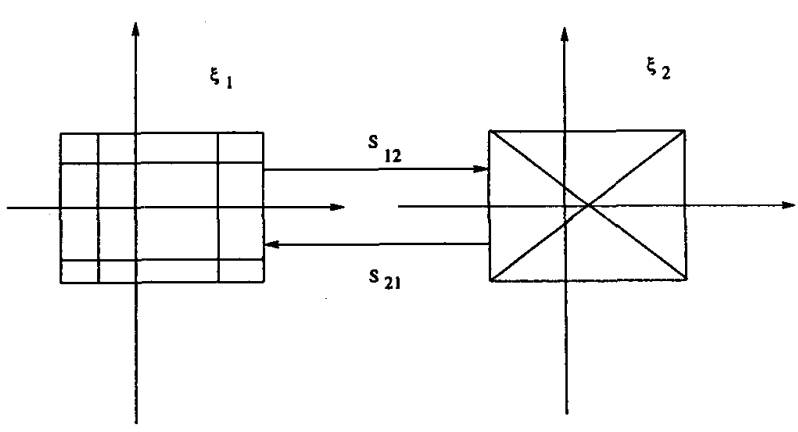

Figure 3: Hybrid Automaton

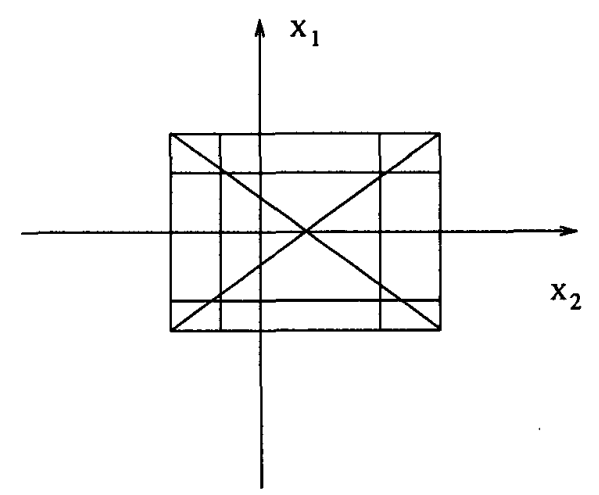

Figure 4: Discrete Event Composition

\section{Step 2. Multilayer Decomposition}

The concept of multilayer cell partition being the key element in the proposed method implies appropriate decomposition of the set $\bigcup_{i=1}^{L} \Omega_{i}$ and studying its properties.

Definition 4.1 The set $\mathbf{E}(\Omega)=\left\{\Omega_{i}\right\}_{i \in\{1,2, \cdots L\}}$ is said to be the outer layer of the set $\Omega$ if the following conditions are satisfied

$\mathbf{E}(\Omega)=\left\{\begin{array}{l}\Omega \text { if } \exists \Omega_{i} \text { s.t. } 0 \in \text { int } \Omega_{i}, \partial \Omega \cap \Omega_{i} \neq \emptyset \\ \left\{\Omega_{i}: \Omega_{i} \cap \partial \Omega \neq \emptyset\right\} \quad \text { otherwise }\end{array}\right.$

Compute recursively

$$
\mathbf{E}_{k}=\mathbf{E}\left(\Omega-\bigcup_{i=0}^{k-1} \mathbf{E}_{i}\right)
$$

where $\mathbf{E}_{0}=\emptyset$. Each set $\mathbf{E}_{k} \neq \emptyset, k=1,2, \ldots$ will be further referred to as the $k$-th layer of $\Omega$. 
Definition 4.2 We say that the system is in the convex multilayer form if the following conditions are satisfied

$$
\Omega-\bigcup_{i=1}^{k} \mathbf{E}_{i}=\operatorname{conv}\left\{\Omega-\bigcup_{i=1}^{k} \mathbf{E}_{i}\right\}, \forall 1 \leq k \leq \bar{k}
$$

where $\bar{k}=\arg \max _{k}\left\{k: k>0, \mathbf{E}_{k} \neq \emptyset\right\}$.

Without loss of generality we further assume that the hybrid system $(2.1),(2.2)$ is in the convex multilayer form. If this is not the case then it is always possible to use a redundant decomposition of $\Omega$ satisfying conditions (4.7) (see Figure 5 and the full version of the paper).

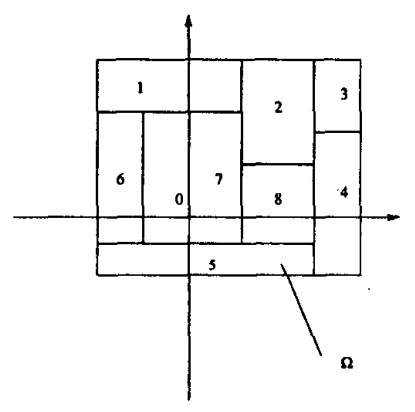

(a)

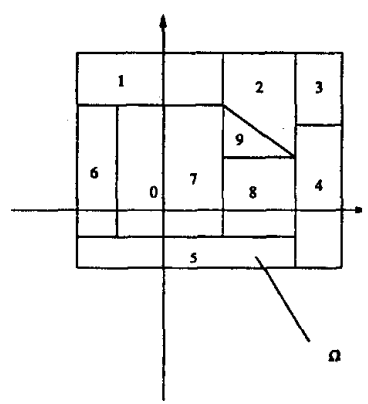

(b)
Figure 5: Convex Multilayer Cell Partition

\section{Stabilizability Analysis}

For a given convex set $\Omega \subset \mathbf{R}^{\mathbf{n}}$ let $\Omega_{\alpha} \subset \mathbf{R}^{\mathbf{n}}$ denote the parameterised set defined as $\Omega_{\alpha}=\{Y: Y=\alpha X, X \in$ $\Omega, \alpha>0\}$. Given a vector $Z \in \mathbf{R}^{\mathbf{n}}$, we say that the set $\Omega_{\alpha}$ is a proper set of $Z$ if $Z \in \partial \Omega_{\alpha}$. We will further use the notation $\Omega_{\alpha}(Z)$ to refer to such sets. Let us introduce the class of positive definite polyhedral Lyapunov functions $^{1} V_{\Omega}(x)=\left\{\alpha: \alpha \geq 0, \Omega_{\alpha}=\Omega_{\alpha}(x)\right\}$

\footnotetext{
${ }^{1}$ a positive definite Lyapunov function $V(x)$ is said to be polyhedral if its level set $B_{r}=\left\{x \in \mathbf{R}^{\mathbf{n}}: \mathbf{V}(\mathbf{x})=\mathbf{r}\right\}$ is a convex polyhedron for all $r \geq 0$, moreover, for any $r_{1} \geq 0, r_{2} \geq 0$,
}

where int $\Omega \neq \varnothing$ and int $\{\cdot\}$ stands for the interior of a set. Having transformed the system to a multilayer convex form (Steps 1,2) we are ready now to start analysing the stabilizability properties of the system with respect to the polyhedral Lyapunov functions $V_{k}(x)$ naturally induced by each layer $\mathbf{E}_{k}$. The main idea behind the analysis is that each cell $\Omega_{k i} \in \mathbf{E}_{k}$ can be analysed separately expecting that certain properties of the cells combined together would guarantee stabilizability of the overall system. In the following description of the evolution of the continuous states within a cell we deliberately omit the indices $i(x)$, that is $f(x(t), \xi(t))=f(x(t), i(x(t)), \xi(t))$. Let $\overline{\mathbf{E}}_{k}$ denote the convex hull of the set $\mathbf{E}_{k}$, that is, $\overline{\mathbf{E}}_{k}=$ conv $\mathbf{E}_{k}$.

\section{Definition 5.1 (Cell Stabilizability)}

A cell $\Omega_{k i} \subseteq \mathbf{E}_{k}$ is said to be stabilizable w.r.t. the Lyapunov function $V_{k}(x)=V_{\tilde{E}_{k}}(x)$ if there exist a positive constant $0<\beta<1$ and a switching index $\xi \in Q$ such that $V_{k}(f(x, \xi))-\beta V_{k}(x) \leq 0$ holds for all $x \in \Omega_{k i}$.

Theorem 5.1 A hybrid system (2.1),(2.2) is stabilizable on $\Omega$ if every cell $\Omega_{k i}$ is stabilizable w.r.t. the Lyapunov function $V_{k}(x)$.

By Theorem 5.1 the stabilizability analysis of the hybrid system is reduced to that of each cell obtained as a result of the convex multilayer decomposition.

\subsection{Step 3. Cell Stabilizability Analysis}

First we recall that the polyhedral Lyapunov function $V_{k}(x)$ is defined as $V_{k}(x)=\max _{1 \leq j \leq s}\left\{c_{j}^{T} x\right\}$ where each vector $c_{j}, j=1,2, \ldots, s$ defines a hyperplane $r_{1} \leq r_{2}$ the following condition holds $B_{r_{2}}=\frac{r_{2}}{r_{1}} B_{r_{1}}$ holds. An alternative description of polyhedral Lyapunov functions can be given in the form $V_{\Omega}(x)=\max _{1 \leq i \leq s}\left\{c_{i}^{T} x\right\}$. See, for example [2] and references therein. 
$c_{j}^{T} x=z_{j}$ bounding the polyhedron $\ddot{\mathbf{E}}_{k}$. The theorems below represent the main results of the paper.

Theorem $5.2 A$ cell $\Omega_{k i} \subseteq \mathbf{E}_{k}, 0 \notin \Omega_{k i}$ is stabilizable w.r.t. the Lyapunov function $V_{k}(x)$ if and only if

$$
\bigcap_{j=1}^{s} I_{j} \neq \varnothing
$$

where

$$
I_{j}=\left\{\xi \in Q: \max _{\alpha \in \Upsilon} \max _{x \in \Omega_{k i} \bigcap\left\{x: V_{k}(x)=\alpha\right\}} \frac{c_{j}^{T} f(x, \xi)}{\alpha}<1\right\},,^{2}
$$

and

$$
\Upsilon_{k i}=\left[\min _{x \in \Omega_{k i}} V_{k}(x), \max _{x \in \Omega_{k i}} V_{k}(x)\right]
$$

Below we show that under certain mild conditions the problem of calculating the sets $I_{j}, j=1, \ldots, s$ is reduced to a standard nonlinear optimization problem.

Theorem 5.3 Let the following conditions be satisfied: (i) The functions $f(x, \xi), \xi \in Q$ are Lipschitz over $\Omega_{k i}$, that is, $\left\|f\left(x_{1}, \xi\right)-f\left(x_{2}, \xi\right)\right\| \leq L\left\|x_{1}-x_{2}\right\|$ holds for some $0<L<\infty$, (ii) $0 \notin \Omega_{k i}$. Then the cell $\Omega_{k i}$ is stabilizable w.r.t. the Lyapunov function $V_{k}(x)$ if and only if there exists a finite number of constants $\alpha_{l}, l=1, \ldots, m, \alpha_{1}=\alpha^{-}=\min _{x \in \Omega_{k i}} V_{k}(x), \alpha_{m}=$ $\alpha^{+}=\max _{x \in \Omega_{k i}} V_{k}(x), \alpha_{s}=\alpha_{s-1}+\left(\alpha^{+}-\alpha^{-}\right) / m$, $\forall 1<s<m$ such that

$$
\bigcap_{j=1}^{s} \bigcap_{l=1}^{m} I_{j l} \neq \varnothing
$$

where

$$
\begin{aligned}
I_{j l}=\{\xi \in Q: & \max _{x \in \Omega_{k i} \bigcap\left\{x: V_{k}(x)=\alpha_{l}\right\}} \frac{c_{j}^{T} f(x, \xi)}{\alpha_{l}} \\
& \left.<1-\frac{L\left\|c_{j}\right\| \delta\left(\alpha_{l}, \alpha_{l+1}\right)}{\alpha_{l}}\right\}
\end{aligned}
$$

${ }^{2}$ For the sake notational simplicity we avoid here the notation $I_{j}\left(\Omega_{k i}\right)$ implying $I_{j}=I_{j}\left(\Omega_{k i}\right)$. and

$$
\delta\left(\alpha_{l}, \alpha_{l+1}\right)=\max _{\left\{x: V_{k}(x)=\alpha_{l}\right\}}\left\|x\left(\frac{\alpha_{l+1}}{\alpha_{l}}-1\right)\right\|
$$

To analyse the case $0 \in \Omega_{k i}$ we note that the last inclusion implies the layer $\mathbf{E}_{k}$ being the last layer in the convex decomposition. Since $\alpha^{-}=0$ the result of Theorem 5.3 can be directly applied by replacing $\alpha^{-}$ by a sufficiently small positive constant. In that case the existence of a sufficiently small attractor centred around the origin can be proved under some additional local stabilizability assumptions (see the full version of the paper).

Remark 5.1 The computational complexity of checking the conditions (5.8),(5.11) depends on the topological properties of the nonlinear system and must be assessed on a case by case basis. We show that piecewise linear hybrid systems of type (2.1),(2.2) allow for significantly simplified procedures for checking the conditions (5.8),(5.11).

The systematic approach to stabilizing control design presented above leads to simple switching control design algorithms presented in the full version of the paper.

\section{Model Example}

Example 6.1 Let us consider the following two discrete state hybrid system. For both discrete states $\xi_{1}$ and $\xi_{2}$ the normal operating domain is specified as $\Omega=\left\{x \in \mathbf{R}^{2}:\left|\mathbf{x}_{1}\right| \leq \mathbf{1},\left|\mathbf{x}_{2}\right| \leq \mathbf{1}\right\}$.

The continuous dynamics corresponding to the first discrete state $\xi_{1}$ is described by

$$
x(t+1)=\left[\begin{array}{ll}
1 & 0 \\
0 & 2.6 e^{\operatorname{sgn}\left(x_{1}+x_{2}\right)\left(x_{1}-x_{2}\right)}
\end{array}\right] x(t)
$$


thus partitioning the normal operating domain $\Omega$ in stable and unstable sub-domains, respectively.

For the discrete state $\xi_{2}$ the domain $\Omega$ is partitioned into three sub-domains by the hyperplanes $x_{1}-x_{2}=0$, $x_{1}+x_{2}=0$ and $x_{1}-3 x_{2}=0$, with the continuous dynamics being piecewise linear

$$
x(t+1)=A_{i(t)} x(t)+F d(t), i(t) \in\{1,2,3\}
$$

where

$$
\begin{aligned}
& A_{1}=\left[\begin{array}{cc}
0.9539 & -0.0361 \\
0.7461 & 0.5161
\end{array}\right], \\
& {\left[\begin{array}{rr}
-0.9000 & 0.2333 \\
-0.2000 & 0.8000
\end{array}\right], \quad A_{3}=\left[\begin{array}{rr}
-1.4 & -0.3 \\
1.3 & 2.4
\end{array}\right]}
\end{aligned}
$$

and the switching logic is defined as

$$
i(t)=\left\{\begin{array}{lll}
1 & \text { if } & x(t) \in \Omega_{21} \\
2 & \text { if } & x(t) \in \Omega_{22} \\
3 & \text { if } & x(t) \in \Omega_{23}
\end{array}\right.
$$

Performing the discrete event composition we obtain a cone type cell partition of the hybrid system depicted in Figure 6. Further analysis resulted in the following stabilizing switching controller

$\Gamma(\xi, \mathbf{x}):\left\{\begin{array}{lll}\xi_{1} \rightarrow \xi_{2} & \text { if } & \xi(t)=\xi_{1} \text { and } x(t) \in \Omega_{1} \cup \Omega_{2} \\ \xi_{2} \rightarrow \xi_{1} & \text { if } \quad \xi(t)=\xi_{2} \text { and } x(t) \in \Omega_{3}\end{array}\right.$

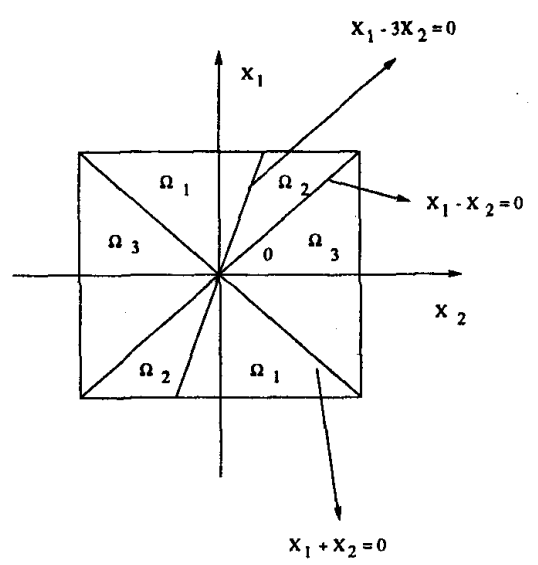

Figure 6: Transformed Hybrid System

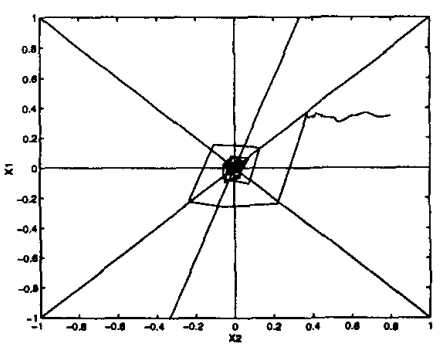

Figure 7: Evolution of the Continuous States

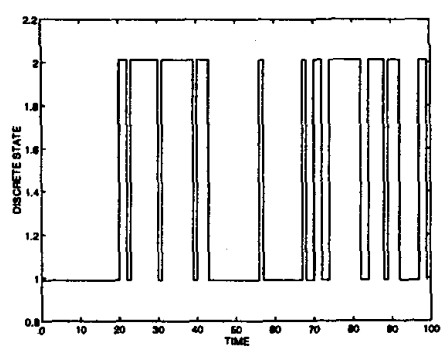

Figure 8: Switching Sequence

The behaviour of the system subject to random uniformly distributed disturbance $d(t): \sup _{t \geq t_{0}}|d(t)|=$ 0.01 and $F=(1,1)^{T}$ is shown in Figures 7,8 .

\section{Conclusions}

In this paper we propose and investigate a new theoretical framework for controlling and analysing a class of linear and nonlinear discrete time hybrid systems.

\section{References}

[1] M. Johansson and A. Rantzer, "Computation of Piecewise Quadratic Lyapunov Functions for Hybrid Systems," Prepr., Depart. of Automatic Control, Lund Institute of Technology, 1996.

[2] A. Polanski, "Lyapunov Function Construction by Linear Programming," IEEE TAC, v. 42, no. 7, pp. 1013-1016, 1997. 